\title{
Recent progress in using laser structured high energy electrode materials for next generation batteries (Conference Presentation) (Withdrawal Notice)
}

Wilhelm Pfleging, Peter Smyrek, Jan-Hendric Rakebrandt, $X$. Chen, Y. Zhang, et al.

Wilhelm Pfleging, Peter Smyrek, Jan-Hendric Rakebrandt, X. Chen, Y. Zhang, Yijing Zheng, "Recent progress in using laser structured high energy electrode materials for next generation batteries (Conference Presentation) (Withdrawal Notice)," Proc. SPIE 10906, Laser-based Micro- and Nanoprocessing XIII, 109061C (7 June 2019); doi: 10.1117/12.2515726

SPIE. Event: SPIE LASE, 2019, San Francisco, California, United States 


\section{Recent progress in using laser structured high energy electrode materials for next generation batteries (Conference Presentation) (Withdrawal Notice)}

Wilhelm Pfleging, ${ }^{1,2}$ Peter Smyrek, ${ }^{1,2}$ Jan-Hendric Rakebrandt, ${ }^{1}$ X. Chen, ${ }^{3}$ Y. Zhang, ${ }^{3}$ Yijing Zheng'

'Karlsruher Institut für Technologie (Germany)

2Karlsruhe Nano Micro Facility (Germany)

3Beijing Univ. of Technology (China)

Proceedings Volume 10906, Laser-based Micro- and Nanoprocessing XIII; $109061 \mathrm{C}$ (2019)

https://doi.org/10.1117/12.2515726

Event: SPIE LASE, 2019, San Francisco, California, United States

Online Publication Date: 4 March 2019

Withdrawn from Publication: 7 June 2019

Publisher's Note: This conference presentation, originally published on 4 March 2019, was withdrawn per author request. 